\title{
Triple-Frequency GPS Precise Point Positioning Ambiguity Resolution Using Dual-Frequency Based IGS Precise Clock Products
}

\author{
Fei Liu and Yang Gao \\ Department of Geomatics Engineering, University of Calgary, Calgary, AB, Canada \\ Correspondence should be addressed to Fei Liu; liu19@ucalgary.ca
}

Received 16 September 2016; Revised 6 January 2017; Accepted 17 January 2017; Published 21 February 2017

Academic Editor: Salvatore Gaglione

Copyright (c) 2017 Fei Liu and Yang Gao. This is an open access article distributed under the Creative Commons Attribution License, which permits unrestricted use, distribution, and reproduction in any medium, provided the original work is properly cited.

\begin{abstract}
With the availability of the third civil signal in the Global Positioning System, triple-frequency Precise Point Positioning ambiguity resolution methods have drawn increasing attention due to significantly reduced convergence time. However, the corresponding triple-frequency based precise clock products are not widely available and adopted by applications. Currently, most precise products are generated based on ionosphere-free combination of dual-frequency L1/L2 signals, which however are not consistent with the triple-frequency ionosphere-free carrier-phase measurements, resulting in inaccurate positioning and unstable float ambiguities. In this study, a GPS triple-frequency PPP ambiguity resolution method is developed using the widely used dual-frequency based clock products. In this method, the interfrequency clock biases between the triple-frequency and dual-frequency ionosphere-free carrier-phase measurements are first estimated and then applied to triple-frequency ionosphere-free carrier-phase measurements to obtain stable float ambiguities. After this, the wide-lane L2/L5 and wide-lane L1/L2 integer property of ambiguities are recovered by estimating the satellite fractional cycle biases. A test using a sparse network is conducted to verify the effectiveness of the method. The results show that the ambiguity resolution can be achieved in minutes even tens of seconds and the positioning accuracy is in decimeter level.
\end{abstract}

\section{Introduction}

With precise satellite orbit and clock products, Precise Point Positioning (PPP) using ionosphere-free (IF) code and carrier-phase observations can achieve centimeter-level accuracy if advanced error calibration models are applied [1]. The main disadvantage of PPP is that it needs significant time to reach convergence. Fast ambiguity resolution (AR) is requested to reduce this convergence time. Ambiguity fixed solutions can also further improve the PPP accuracy. Several PPP integer ambiguity resolution methods have been explored and developed in recent years [2-4]. However, it still takes a few tens of minutes to obtain reliable ambiguity resolution (AR) if only with dual-frequency observations. This is because large noise of code measurements leads to long time smoothing with Melbourne-Wübbena (MW) measurement combination $[5,6]$. Moreover, the narrow-lane (NL) ambiguities need more than ten minutes to be fixed due to short wavelength [7].

At present, the third Global Positioning System (GPS) civil signal L5 is available with the launch of the latest Block IIF satellites, which enables more flexible ambiguity resolution strategies. Triple-frequency PPP AR can be achieved faster with longer wavelength, which has been studied by researchers [4, 8-10]. Gu et al. [9] verified the effectiveness of triple-frequency PPP using BeiDou datasets. For GPS triplefrequency PPP, Geng and Bock [8] fixed the ambiguities in each frequency and proved higher efficiency using simulated GPS datasets; Wang [10] only fixed the extra-wide-lane (EWL) and wide-lane (WL) ambiguities with a windowed phase smoothing phase technique to reduce the large noise of triple-frequency IF measurements. However, both GPS triple-frequency PPP AR researches mentioned above used simulated datasets, which would not suffer the inconsistency 
between the dual-frequency based precise clock products and the triple-frequency IF carrier-phase measurements. Very few researchers implemented the GPS triple-frequency PPP with measured datasets. Laurichesse [11] implemented the GPS triple-frequency PPP AR with integer clock products applicable for each single frequency carrier-phase measurements, which is however not widely adopted by applications yet. If widely applied dual-frequency based precise clock products are used in GPS triple-frequency PPP with observed data, there is obvious inconsistency between the dual-frequency based precise clock products and the triplefrequency IF measurements. This is because unlike the interfrequency clock biases (IFCBs) between dual-frequency IF code and phase measurement, which is stable over certain time [3], the IFCB between dual-frequency IF code and triple-frequency IF phase measurement varies up to meters over time [12, 13]. In this paper, GPS triple-frequency PPP AR method using widely used dual-frequency based precise clock products and measured datasets is proposed. In this method, the IFCBs between the dual-frequency and triple-frequency IF carrier-phase measurements are first estimated and then applied to triple-frequency IF carrier-phase measurements before using triple-frequency IF carrier-phase measurements in PPP. In this way, the widely applied dual-frequency precise clock products can be used in triple-frequency PPP. After applying the estimated IFCBs, the satellite fractional cycle biases (FCBs) can be estimated and used to recover the integer property of ambiguities. In other words, compared to dualfrequency PPP, in addition to FCBs, IFCBs between dualfrequency and triple-frequency IF carrier-phase measurements are also broadcasted to users to implement the triplefrequency GPS PPP AR.

Montenbruck et al. [13] and Li et al. [12] provided solutions for estimating L1/L2 and L1/L5 IFCBs, which can be also applied for estimating L1/L2 and triple-frequency IF carrier-phase IFCBs. Then, the triple-frequency GPS PPP AR using dual-frequency based IGS precise clock products and observed datasets can be conducted. Specifically, singledifference between-satellite PPP AR fixing only EWL and WL ambiguities is implemented. Due to limited number of GPS satellites with triple-frequency observables, dual-frequency and triple-frequency IF carrier-phase measurements are applied together to obtain PPP solutions. Datasets of one sparse network with eight reference stations in Europe are used to first estimate the IFCBs. The extra-wide-lane (EWL) and wide-lane (WL) FCBs can be generated by the reference stations in the networks after applying the estimated IFCBs. Both IFCB and FCB products have to be sent to test user stations. At the test user stations, the IFCBs are used to obtain stable float solutions. Then, after applying the estimated EWL and WL FCBs, the EWL ambiguities can be fixed instantaneously with the Melbourne-Wübbena combination while the WL ambiguities can be determined by the LAMBDA method [14]. Different from dual-frequency PPP AR which has to resolve the narrow-lane (NL) ambiguity, L1/L2 widelane ambiguity with valid wavelength of $3.4 \mathrm{~m}$ needs to be fixed in triple-frequency PPP AR, which means that fast AR can be achieved with longer wavelength. The paper is organized as follows. In Section 2, the procedures of dualfrequency single-difference PPP ambiguity resolution using dual-frequency based IGS precise products are presented. Section 3 introduces the problem of implementing triplefrequency PPP ambiguity resolution using the method in Section 2 and provides the solution. The test results and conclusions are shown in Sections 4 and 5, respectively.

\section{Dual-Frequency PPP AR Using Dual-Frequency Based Precise Products}

The dual-frequency undifferenced ionosphere-free (IF) combination of code and phase measurements after applying each error model (e.g., Sagnac effect, relative effect) can be expressed as

$$
\begin{aligned}
P_{\text {dual }}= & \frac{f_{1}^{2}}{f_{1}^{2}-f_{2}^{2}} P_{1}-\frac{f_{2}^{2}}{f_{1}^{2}-f_{2}^{2}} P_{2} \\
= & \rho+\left(c d t^{r}+b_{P_{\text {dual }}}^{r}\right)-\left(c d t^{s}+b_{P_{\text {dual }}}^{s}\right)+T \\
& +\varepsilon\left(P_{\text {dual }}\right) \\
= & \rho+c d t_{P_{\text {dual }}}^{r}-c d t_{P_{\text {dual }}}^{s}+T+\varepsilon\left(P_{\text {dual }}\right) \\
\Phi_{\text {dual }}= & \frac{f_{1}^{2}}{f_{1}^{2}-f_{2}^{2}} \Phi_{1}-\frac{f_{2}^{2}}{f_{1}^{2}-f_{2}^{2}} \Phi_{2} \\
= & \rho+\left(c d t^{r}+b_{\Phi_{\text {dual }}}^{r}\right)-\left(c d t^{s}+b_{\Phi_{\text {dual }}}^{s}\right)+T \\
& +\lambda_{\text {dual }} N_{\text {dual }}+\varepsilon\left(\Phi_{\text {dual }}\right) \\
= & \rho+c d t_{\Phi_{\text {dual }}^{r}}^{r}-c d t_{\Phi_{\text {dual }}}^{s}+T+\lambda_{\text {dual }} N_{\text {dual }} \\
& +\varepsilon\left(\Phi_{\text {dual }}\right) \\
c d t_{P_{\text {dual }}}^{r}= & c d t^{r}+b_{P_{\text {dual }}}^{r} \\
c d t_{P_{\text {dual }}}^{s}= & c d t^{s}+b_{P_{\text {dual }}}^{s} \\
c d t_{\Phi_{\text {dual }}}^{r}= & c d t^{r}+b_{\Phi_{\text {dual }}}^{r} \\
c d t_{\Phi_{\text {dual }}}^{s}= & c d t^{s}+b_{\Phi_{\text {dual }}}^{s}
\end{aligned}
$$

where $P_{\text {dual }}$ and $\Phi_{\text {dual }}$ represent the undifferenced dualfrequency ionosphere-free (IF) code and carrier-phase measurements $(\mathrm{m}), f_{1}$ and $f_{2}$ represent the $\mathrm{L} 1$ and $\mathrm{L} 2$ frequencies, $P_{1}, P_{2}$ and $\Phi_{1}, \Phi_{2}$ are L1 and L 2 code and phase measurements in unit of meter, respectively, $\rho$ is the geometric range between receiver and satellite, $c$ is the speed of light in vacuum, $c d t_{P_{\text {dual }}}^{s}$ (satellite code clock error), $c d t_{\Phi_{\text {dual }}}^{s}$ (satellite phase clock error), $c d t_{P_{\text {dual }}}^{r}$ (receiver code clock error), and $c d t_{\Phi_{\text {dual }}}^{r}$ (receiver phase clock error) are a function of the actual satellite clock error $d t^{s}$, receiver clock error $d t^{r}$, satellite dual-frequency IF code and phase biases, $b_{P_{\text {dual }}}^{s}, b_{\Phi_{\text {dual }}}^{s}$, and receiver dual-frequency IF code and phase biases $b_{P_{\text {dual }}}^{r}, b_{\Phi_{\text {dual }}}^{r}$. $T$ is the tropospheric delay, $\varepsilon\left(P_{\text {dual }}\right)$ and $\varepsilon\left(\Phi_{\text {dual }}\right)$ are the noise including multipath of dual-frequency IF code and carrierphase measurements, and $\lambda_{\text {dual }}$ and $N_{\text {dual }}$ represent the 
wavelength and ambiguity of dual-frequency IF combination, which is given as

$$
\begin{aligned}
\lambda_{\text {dual }} N_{\text {dual }} & =\frac{f_{1}^{2} \lambda_{1} N_{1}-f_{2}^{2} \lambda_{2} N_{2}}{f_{1}^{2}-f_{2}^{2}} \\
& =\lambda_{\mathrm{NL}} N_{1}+\frac{f_{2}}{f_{1}+f_{2}} \lambda_{\mathrm{WL}} N_{\mathrm{WL}},
\end{aligned}
$$

where $N_{1}$ and $N_{2}$ are the integer ambiguities of L1 and L2 frequencies, $N_{\mathrm{WL}}$ and $N_{\mathrm{NL}}$ are the wide-lane and narrowlane integer ambiguities formed by $N_{1}$ and $N_{2}$, and $\lambda_{\mathrm{WL}}$ and $\lambda_{\mathrm{NL}}$ represent the wavelength of wide-lane and narrow-lane combination, respectively. In float PPP solution, the satellite code clock error $c d t_{P_{\text {dual }}}$ can be provided by IGS precise clock products. The position, tropospheric delay, and receiver code clock error $c d t_{P_{\text {dual }}}^{r}$, together with the float ambiguities are going to be estimated.

In this study, PPP AR is implemented using the strategy proposed by Ge et al. [3] where single-difference (SD) FCBs are applied to recover the single-difference ambiguity integer property. The single-difference eliminates the receiver clock and bias. Here the dual-frequency PPP AR is introduced first to better illustrate the triple-frequency PPP AR. The singledifference dual-frequency observation model after applying various error correction models can be rewritten as

$$
\begin{aligned}
\nabla P_{\text {dual }}= & \frac{f_{1}^{2}}{f_{1}^{2}-f_{2}^{2}} \nabla P_{1}-\frac{f_{2}^{2}}{f_{1}^{2}-f_{2}^{2}} \nabla P_{2} \\
= & \nabla \rho-\nabla c d t_{P_{\text {dual }}}^{s}+\nabla T+\varepsilon\left(\nabla P_{\text {dual }}\right) \\
\nabla \Phi_{\text {dual }}= & \frac{f_{1}^{2}}{f_{1}^{2}-f_{2}^{2}} \nabla \Phi_{1}-\frac{f_{2}^{2}}{f_{1}^{2}-f_{2}^{2}} \nabla \Phi_{2} \\
= & \nabla \rho-c \nabla d t_{\Phi_{\text {dual }}}^{s}+\nabla T+\lambda_{\text {dual }} \nabla N_{\text {dual }} \\
& +\varepsilon\left(\nabla \Phi_{\text {dual }}\right) \\
= & \nabla \rho-c \nabla d t_{P_{\text {dual }}}^{s}+\nabla T+\nabla A_{\text {dual }} \\
\nabla A_{\text {dual }}= & c \nabla d t_{P_{\text {dual }}}^{s}-c \nabla d t_{\Phi_{\text {dual }}}^{s}+\lambda_{\text {dual }} \nabla N_{\text {dual }} \\
& +\varepsilon\left(\nabla \Phi_{\text {dual }}\right) \\
= & \nabla b_{P_{\text {dual }}^{s}}^{s}-\nabla b_{\Phi_{\text {dual }}}^{s}+\lambda_{\mathrm{NL}} \nabla N_{1} \\
& +\frac{f_{2}}{f_{1}+f_{2}} \lambda_{\mathrm{WL}} \nabla N_{\mathrm{WL}}+\varepsilon\left(\nabla \Phi_{\text {dual }}\right),
\end{aligned}
$$

where $\nabla$ is the single-difference between-satellite operator. $\nabla A_{\text {dual }}$ is the single-difference float ambiguity. $\nabla N_{\mathrm{WL}}$ and $\nabla N_{\mathrm{NL}}$ are the single-difference wide-lane and narrow-lane integer ambiguities. In this section, because signals in only two frequencies are involved, the wide-lane and narrow-lane represent the corresponding combination of L1 and L2. In the next section, the wide-lane combinations will be specified. It can be seen that the IF ambiguities can be resolved by fixing the wide-lane and L1 ambiguities sequentially, which is illustrated as follows.
The single-difference between-satellite Melbourne-Wübbena (MW) combination can be formed as

$$
\begin{aligned}
\nabla A_{\mathrm{MW}}= & \nabla \Phi_{\mathrm{WL}}-\nabla P_{\mathrm{NL}} \\
= & \lambda_{\mathrm{WL}} \nabla N_{\mathrm{WL}}-\nabla b_{\mathrm{MW}}^{s}+\varepsilon\left(\nabla A_{\mathrm{MW}}\right) \\
\nabla b_{\mathrm{MW}}^{s}= & \left(\alpha_{\mathrm{WL}} \nabla b_{\Phi 1}^{s}+\beta_{\mathrm{WL}} \nabla b_{\Phi 2}^{s}\right) \\
& -\left(\alpha_{\mathrm{NL}} \nabla b_{P 1}^{s}+\beta_{\mathrm{NL}} \nabla b_{P 2}^{s}\right) \\
\alpha_{\mathrm{WL}}= & \frac{f_{1}}{f_{1}-f_{2}} \\
\beta_{\mathrm{WL}}= & -\frac{f_{2}}{f_{1}-f_{2}} \\
\alpha_{\mathrm{NL}}= & \frac{f_{1}}{f_{1}+f_{2}} \\
\beta_{\mathrm{NL}}= & \frac{f_{2}}{f_{1}+f_{2}},
\end{aligned}
$$

where $\nabla A_{\mathrm{MW}}$ is the single-difference MW combination, WL represents wide-lane combination while NL means narrowlane combination, $b_{\mathrm{MW}}^{s}$ is the satellite MW bias, and $b_{\Phi 1}^{s}$, $b_{P 1}^{s}, b_{\Phi 2}^{s}$, and $b_{P 2}^{s}$ represent the satellite phase and code biases of L1 and L2 frequencies. $\alpha_{\mathrm{WL}}, \beta_{\mathrm{WL}}, \alpha_{\mathrm{NL}}$, and $\beta_{\mathrm{NL}}$ are the corresponding wide-lane and narrow-lane coefficients. The single-difference satellite wide-lane FCB at a receiver in unit of meter can be achieved by

$$
\begin{aligned}
& \nabla N_{\mathrm{WL}}=\left\langle\frac{\nabla A_{\mathrm{MW}}}{\lambda_{\mathrm{WL}}}\right\rangle \\
& \nabla b_{\mathrm{MW}}^{s}=\nabla A_{\mathrm{MW}}-\lambda_{\mathrm{WL}} \nabla N_{\mathrm{WL}},
\end{aligned}
$$

where $\langle *\rangle$ denotes rounding of the real value to the nearest integer value. The wide-lane FCB correction with high precision can be calculated by averaging the corrections obtained in a network. For users, after applying the averaged widelane FCBs, the wide-lane ambiguities can be calculated by rounding of the $\mathrm{WL}$ ambiguity real value to the nearest integer value.

After solving the wide-lane ambiguities, if the receiver coordinate is already known, (3) can be rewritten with known parameters on the left side and unknowns on the right side as

$$
\begin{aligned}
& \nabla \Phi_{\text {dual }}-\frac{f_{2}}{f_{1}+f_{2}} \lambda_{\mathrm{WL}} \nabla N_{\mathrm{WL}}-\nabla \rho+c \nabla d t_{P_{\text {dual }}}^{s} \\
& =\nabla T+\lambda_{\mathrm{NL}} \nabla N_{1}+\nabla b_{P_{\text {dual }}}^{s}-\nabla b_{\Phi_{\text {dual }}}^{s}+\varepsilon\left(\nabla \Phi_{\text {dual }}\right) \\
& =\nabla T+\nabla A_{1} \\
& \nabla A_{1}=\lambda_{\mathrm{NL}} \nabla N_{1}+\nabla b_{P_{\text {dual }}}^{s}-\nabla b_{\Phi_{\text {dual }}}^{s}+\varepsilon\left(\nabla \Phi_{\text {dual }}\right),
\end{aligned}
$$


where $\nabla A_{1}$ is the float ambiguity of $N_{1}$. Similar to calculation of wide-lane FCB corrections, $N_{1}$ FCB corrections at a receiver in a network after convergence can be calculated by

$$
\begin{aligned}
& \nabla N_{1}=\left\langle\frac{\nabla A_{1}}{\lambda_{\mathrm{NL}}}\right\rangle \\
& \nabla b_{N_{1}}^{s}=\nabla b_{P_{\text {dual }}^{s}}^{s}-\nabla b_{\Phi_{\text {dual }}^{s}}^{s}=\nabla A_{1}-\lambda_{\mathrm{NL}} \nabla N_{1} .
\end{aligned}
$$

By averaging the calculated $N_{1}$ FCB corrections from multiple receivers in a network, a precise value can be achieved. When users receive and apply the $N_{1}$ FCBs in the carrierphase measurements, the integer property of $N_{1}$ ambiguities can be recovered. Then, $N_{1}$ can be resolved by applying LAMBDA method.

\section{Triple-Frequency PPP AR Using Dual-Frequency Based Precise Products}

According to Wang [10] and Feng [15], undifferenced triplefrequency IF carrier-phase observation model after applying error models can be written as

$$
\begin{aligned}
\Phi_{\text {triple }}= & \frac{f_{1}}{f_{1}-f_{5}} \Phi_{\mathrm{WL}_{\mathrm{LL1L2}}}-\frac{f_{5}}{f_{1}-f_{5}} \Phi_{\mathrm{WL}_{\mathrm{L} 215}} \\
= & \rho+\left(c d t^{r}+b_{\Phi_{\text {triple }}^{r}}^{r}\right)-\left(c d t^{s}+b_{\Phi_{\text {triple }}}^{s}\right)+T \\
& +\lambda_{\text {triple }} N_{\text {triple }}+\varepsilon\left(\Phi_{\text {triple }}\right) \\
= & \rho+c d t_{\Phi_{\text {triple }}}^{r}-c d t_{\Phi_{\text {triple }}}^{s}+T+\lambda_{\text {triple }} N_{\text {triple }} \\
& +\varepsilon\left(\Phi_{\text {triple }}\right),
\end{aligned}
$$

where $\Phi_{\text {triple }}$ is the undifferenced triple-frequency IF carrierphase measurement, $\Phi_{\mathrm{WL}_{\mathrm{L11}}, 2}$ is the wide-lane L1 and L2 carrier-phase measurement, $\Phi_{\mathrm{WL}_{\mathrm{I} 215}}$ is the wide-lane L2 and L5 carrier-phase measurement, $c d t_{\Phi_{\text {triple }}}^{s}$ (satellite phase clock error) and $c d t_{\Phi_{\text {triple }}}^{r}$ (receiver phase clock error) are a function of the actual satellite clock error $d t^{s}$, receiver clock error $d t^{r}$, and satellite and receiver triple-frequency IF phase biases $b_{\Phi_{\text {triple }}}^{s}, b_{\Phi_{\text {triple }}}^{r} \cdot \lambda_{\text {triple }}$ and $N_{\text {triple }}$ represent the wavelength and ambiguity of triple-frequency IF combination, which is given as

$$
\begin{aligned}
\lambda_{\text {triple }} N_{\text {triple }}= & \frac{f_{1}}{f_{1}-f_{5}} \lambda_{\mathrm{WL}_{\mathrm{L} 1122}} N_{\mathrm{WL}_{\mathrm{LIL2} 2}} \\
& -\frac{f_{5}}{f_{1}-f_{5}} \lambda_{\mathrm{WL}_{\mathrm{L} 2155}} N_{\mathrm{WL}_{\mathrm{L} 215}} .
\end{aligned}
$$

Similar to (3), after single-difference between satellites, the observation equation can be rewritten as

$$
\begin{aligned}
& \nabla \Phi_{\text {triple }}=\frac{f_{1}}{f_{1}-f_{5}} \nabla \Phi_{\mathrm{WL}_{\mathrm{L} 1 \mathrm{~L} 2}}-\frac{f_{5}}{f_{1}-f_{5}} \nabla \Phi_{\mathrm{WL}_{\mathrm{L} 2 \mathrm{~L}, 5}} \\
& =\nabla \rho-c \nabla d t_{\Phi_{\text {triple }}}^{s}+\nabla T+\lambda_{\text {triple }} \nabla N_{\text {triple }} \\
& +\varepsilon\left(\nabla \Phi_{\text {triple }}\right) \\
& =\nabla \rho-c \nabla d t_{P_{\text {dual }}}^{s}+c \nabla d t_{P_{\text {dual }}}^{s}-c \nabla d t_{\Phi_{\text {triple }}}^{s}+\nabla T \\
& +\lambda_{\text {triple }} \nabla N_{\text {triple }}+\varepsilon\left(\nabla \Phi_{\text {triple }}\right) \\
& =\nabla \rho-c \nabla d t_{P_{\text {dual }}}^{s}+\nabla T+\nabla A_{\text {triple }} \\
& \nabla A_{\text {triple }}=c \nabla d t_{P_{\text {dual }}}^{s}-c \nabla d t_{\Phi_{\text {triple }}}^{s}+\lambda_{\text {triple }} \nabla N_{\text {triple }} \\
& +\varepsilon\left(\nabla \Phi_{\text {triple }}\right) \\
& =\nabla b_{P_{\text {dual }}}^{s}-\nabla b_{\Phi_{\text {triple }}}^{s}+\frac{f_{1}}{f_{1}-f_{5}} \lambda_{\mathrm{WL}_{\mathrm{LL12}}} N_{\mathrm{WL}_{\mathrm{LL1} 2}} \\
& -\frac{f_{5}}{f_{1}-f_{5}} \lambda_{\mathrm{WL}_{\mathrm{L} 2 \mathrm{~L} 5}} N_{\mathrm{WL}_{\mathrm{L} 2 \mathrm{~L} 5}}+\varepsilon\left(\nabla \Phi_{\text {triple }}\right) \text {, }
\end{aligned}
$$

where $\nabla$ is the single-difference between-satellite operator. $c d t_{P_{\text {dual }}}^{s}$ can be provided by IGS precise clock product and $\nabla A_{\text {triple }}$ is the single-difference triple-frequency float ambiguity.

One issue in triple-frequency PPP AR is that, unlike the dual-frequency PPP AR in which $\nabla b_{P_{\text {dual }}}^{s}-\nabla b_{\Phi_{\text {dual }}}^{s}$ is relatively stable over certain time [3], triple-frequency PPP suffers a totally different situation where the variation of the singledifference ambiguities using dual-frequency based precise products can be up to meters [12,13]. This is caused by the instability between satellite dual-frequency IF code bias and satellite triple-frequency IF phase bias, namely, $\nabla b_{P_{\text {dual }}}^{s}-$ $\nabla b_{\Phi_{\text {triple }}}^{s}$. To implement PPP AR, one prerequisite is to obtain relatively stable float ambiguities. Then, after recovering the integer property of ambiguities, integer ambiguity resolution can be achieved. Since the stability of $\nabla b_{P_{\text {dual }}}^{s}-\nabla b_{\Phi_{\text {dual }}}^{s}$ has been proved, to obtain relative stable triple-frequency IF float ambiguities, one straightforward way is to recover $\nabla b_{\Phi_{\text {dual }}}^{s}$ after correcting the difference between $\nabla b_{\Phi_{\text {dual }}}^{s}$ and $\nabla b_{\Phi_{\text {triple }}^{s}}^{s}$, namely, transforming $\nabla b_{P_{\text {dual }}}^{s}-\nabla b_{\Phi_{\text {triple }}}^{s}$ to $\nabla b_{P_{\text {dual }}}^{s}-\nabla b_{\Phi_{\text {dual }}}^{s}$

3.1. Interfrequency Clock Bias (IFCB) Estimation. As mentioned above, the IFCB $\nabla b_{P_{\text {dual }}}^{s}-\nabla b_{\Phi_{\text {triple }}}^{s}$ is unstable, which is absorbed into float ambiguities, resulting in incorrect position estimation and unstable float ambiguity. In order to transform $\nabla b_{P_{\text {dual }}}^{s}-\nabla b_{\Phi_{\text {triple }}}^{s}$ to $\nabla b_{P_{\text {dual }}}^{s}-\nabla b_{\Phi_{\text {dual }}}^{s}$, the difference between $\nabla b_{\Phi_{\text {triple }}}^{s}$ and $\nabla b_{\Phi_{\text {dual }}}^{s}$ has to be estimated. According to Montenbruck et al. [13] and Li et al. [12], the IFCBs can be obtained based on the difference between the two ionosphere-free phase combinations (L1/L2/L5-minus$\mathrm{L} 1 / \mathrm{L} 2)$ since the ionosphere impact is greatly reduced and 
other nondispersive errors contained in the observations can be eliminated. One thing needs to be mentioned is that the L2 phase center offset (PCO) and phase center variation (PCV) is used to correct for L5 due to similar frequency and unavailability of L5 PCO and PCV. To improve the computation efficiency, Li et al. [12] adopted two strategies namely epoch-differenced (ED), and satellite-differenced and epoch-differenced (SDED) method to calculate the IFCB. Difference between epochs removes the ambiguities while difference between satellites eliminates the contribution of receiver IFCB. The SDED method is applied to calculate the IFCB between dual-frequency and triple-frequency IF phase measurements in this work. IFCB in this section refers to the single-difference between-satellite IFCB. In SDED method, IFCB can be obtained by adding all the IFCB difference between two consecutive epochs from the beginning and the IFCB value at the initial epoch. The specific procedures are presented as follows.

To calculate the IFCB, the difference between the single-difference between-satellite dual-frequency and triplefrequency IF carrier-phase measurements using (3) and (10) can be formed as

$$
\begin{aligned}
\nabla \Phi_{\text {dual }}-\nabla \Phi_{\text {triple }}= & \nabla A_{\text {dual }}-\nabla A_{\text {triple }} \\
= & \nabla b_{\Phi_{\text {triple }}}^{s}-\nabla b_{\Phi_{\text {dual }}}^{s}+\lambda_{\text {dual }} \nabla N_{\text {dual }} \\
& -\lambda_{\text {triple }} \nabla N_{\text {triple }},
\end{aligned}
$$

where $\nabla b_{\Phi_{\text {triple }}}^{s}-\nabla b_{\Phi_{\text {dual }}}^{s}$ is the IFCB between triple-frequency and dual-frequency IF phase measurements. As can be seen from (11), the variation of IFCB is reflected on the variation of difference between triple-frequency and dual-frequency IF phase measurements if no cycle slip occurs. To calculate the IFCB, the constant $\lambda_{\text {dual }} \nabla N_{\text {dual }}-\lambda_{\text {triple }} \nabla N_{\text {triple }}$ needs to be eliminated, which can be achieved by calculating the difference between two consecutive epochs, given by

$$
\begin{aligned}
d & =\nabla \Phi_{\text {dual }}-\nabla \Phi_{\text {triple }} \\
\Delta d & =d_{k}-d_{k-1} \\
& =\left(\nabla b_{\Phi_{\text {triple }}}^{s}-\nabla b_{\Phi_{\text {dual }}}^{s}\right)_{k}-\left(\nabla b_{\Phi_{\text {triple }}}^{s}-\nabla b_{\Phi_{\text {dual }}}^{s}\right)_{k-1},
\end{aligned}
$$

where $d$ is the difference between the single-difference dualfrequency and triple-frequency IF phase measurements, $d_{k}$ is the $d$ at epoch $k$, and $\Delta d$ is the difference of $d$ at two consecutive epochs. It can be seen from (12) that $\Delta d$ is the change of IFCB between two consecutive epochs. In order to improve the redundancy to calculate $\Delta d$, datasets of a network are usually utilized to calculate the elevation-angledependent weighted average $\Delta d$ as

$$
\begin{aligned}
\Delta d & =\sum \frac{p_{i} \Delta d_{i}}{\sum p_{i}}, \\
p_{i} & =\frac{\sin \theta_{i} \sin \theta_{r i}}{\sqrt{\sin ^{2} \theta_{i}+\sin ^{2} \theta_{r i}}},
\end{aligned}
$$

where $\Delta d_{i}$ is $\Delta d$ obtained at $i$ station in the network, $\theta_{i}$ is the corresponding satellite elevation angle at $i$ station, $\theta_{r i}$ represents the reference satellite elevation angle at $i$ station, and $p_{i}$ represents the weight of $\Delta d$ at $i$ station.

The IFCB at any epoch can be calculated by adding $\Delta d$ from the beginning and the IFCB at the initial epoch, expressed as

$$
\delta_{k}=\delta_{0}+\sum \Delta d
$$

where $\delta_{k}$ is the IFCB at epoch $k$ and $\delta_{0}$ is the IFCB at the initial epoch, which can be set as arbitrary value.

So far, $\nabla b_{\Phi_{\text {triple }}}^{s}-\nabla b_{\Phi_{\text {dual }}}^{s}$ can be obtained, which can be applied as another correction to triple-frequency IF carrierphase measurements as $\nabla \Phi_{\text {triple }}+\delta_{k}$. One thing needs to be mentioned is that there is a constant bias between the IFCB calculated and the actual value shown as below

$$
a=\left(\nabla b_{\Phi_{\text {triple }}}^{s}-\nabla b_{\Phi_{\text {dual }}}^{s}\right)_{k}-\delta_{k}
$$

This is because the IFCB at the initial epoch is assumed to be arbitrary value. However, this would not affect integer ambiguity resolution because the majority part of the constant bias only changes the integer values of WL ambiguities while the less than one cycle part will be treated as part of the FCB corrections.

3.2. Ambiguity Resolution. After obtaining the IFCBs, relative stable float ambiguities can be achieved. The next step is to recover the integer property of ambiguities, which is discussed in this part. From (10), it can be seen that the triple-frequency IF ambiguity resolution can be achieved by resolving the wide-lane L2/L5 and wide-lane L1/L2 integer ambiguities sequentially. Since the wavelength of WL L2/L5 is as long as $5.86 \mathrm{~m}$, it will be called extra-wide-lane (EWL) and WL refers to wide-lane of L1 and L2 from now on. Similar to (4) and (5), EWL FCBs at a receiver can be determined by forming MW combination of L2 and L5 measurements as

$$
\begin{aligned}
\nabla A_{\mathrm{MW}_{\mathrm{L} 2 \mathrm{~L} 5}=} & \nabla \Phi_{\mathrm{WL}_{\mathrm{L} 2 \mathrm{~L} 5}}-\nabla P_{\mathrm{NL}_{\mathrm{L} 2 \mathrm{~L} 5}} \\
= & \lambda_{\mathrm{WL}_{\mathrm{L} 2 \mathrm{~L} 5}} \nabla N_{\mathrm{WL}_{\mathrm{L} 2 \mathrm{~L} 5}}-\nabla b_{\mathrm{MW}_{\mathrm{L} 2 \mathrm{~L} 5}} \\
& +\varepsilon\left(\nabla A_{\mathrm{MW}_{\mathrm{L} 2 \mathrm{~L} 5}}\right) \\
\nabla b_{\mathrm{MW}}^{s}= & \left(\alpha_{\mathrm{WL}} \nabla b_{\Phi 2}^{s}+\beta_{\mathrm{WL}} \nabla b_{\Phi 5}^{s}\right) \\
& -\left(\alpha_{\mathrm{NL}} \nabla b_{P 2}^{s}+\beta_{\mathrm{NL}} \nabla b_{P 5}^{s}\right) \\
\alpha_{\mathrm{WL}}= & \frac{f_{1}}{f_{1}-f_{2}} \\
\beta_{\mathrm{WL}}= & -\frac{f_{2}}{f_{1}-f_{2}} \\
\alpha_{\mathrm{NL}}= & \frac{f_{1}}{f_{1}+f_{2}} \\
\beta_{\mathrm{NL}}= & \frac{f_{2}}{f_{1}+f_{2}}
\end{aligned}
$$




$$
\begin{aligned}
\nabla N_{\mathrm{MW}_{\mathrm{L} 2 \mathrm{~L} 5}} & =\left\langle\frac{\nabla A_{\mathrm{MW}_{\mathrm{L} 2 \mathrm{~L} 5}}}{\lambda_{\mathrm{WL}_{\mathrm{L} 2 \mathrm{~L} 5}}}\right\rangle \\
\nabla b_{\mathrm{MW}_{\mathrm{L} 2 \mathrm{~L} 5}}^{s} & =\nabla A_{\mathrm{MW}_{\mathrm{L} 2 \mathrm{~L} 5}}-\lambda_{\mathrm{WL}_{\mathrm{L} 2 \mathrm{~L} 5}} \nabla N_{\mathrm{WL}_{\mathrm{L} 2 \mathrm{~L} 5}} .
\end{aligned}
$$

By averaging the satellite EWL FCBs obtained by the receivers in the network, precise EWL FCBs can be achieved and broadcasted to users. After applying the EWL FCBs, the EWL integer ambiguity can be obtained by rounding the realvalued EWL ambiguity to its nearest integer value.

After solving EWL ambiguities, similar to (6), the WL FCBs can be obtained by

$$
\begin{aligned}
& \nabla \Phi_{\text {triple }}-\nabla \rho+\frac{f_{5}}{f_{1}-f_{5}} \lambda_{\mathrm{WL}_{\mathrm{L} 2 \mathrm{~L} 5}} N_{\mathrm{WL}_{\mathrm{L} 2 \mathrm{~L} 5}}+c \nabla d t_{P_{\text {dual }}}^{s}+\delta \\
& =\nabla T+\nabla b_{P_{\text {dual }}^{s}}^{s}-\nabla b_{\Phi_{\text {dual }}}^{s}+b_{a} \\
& \quad+\frac{f_{1}}{f_{1}-f_{5}} \lambda_{\mathrm{WL}_{\mathrm{L} 1 \mathrm{~L} 2}} \widetilde{N}_{\mathrm{WL}_{\mathrm{L} 1 \mathrm{~L} 2}}+\varepsilon\left(\nabla \Phi_{\text {dual }}\right) \\
& =\nabla T+\nabla A_{\mathrm{WL}}
\end{aligned}
$$

$\nabla A_{\mathrm{WL}}$

$$
\begin{aligned}
= & \nabla b_{P_{\text {dual }}}^{s}-\nabla b_{\Phi_{\text {dual }}}^{s}+b_{a}+\frac{f_{1}}{f_{1}-f_{5}} \lambda_{\mathrm{WL}_{\mathrm{L} 1 \mathrm{~L} 2}} \widetilde{N}_{\mathrm{WL}_{\mathrm{L} 1 \mathrm{~L} 2}} \\
& +\varepsilon\left(\nabla \Phi_{\text {dual }}\right),
\end{aligned}
$$

where $\nabla A_{\mathrm{WL}}$ is the float WL ambiguity, $\delta$ is the IFCB obtained by (14), $b_{a}$ is the less than one cycle part of the bias $a$ in (15), and $\widetilde{N}_{\mathrm{WL}_{\mathrm{L} 1 \mathrm{~L} 2}}$ is the pseudo WL ambiguities after absorbing the majority part of bias $a$ in (15). Due to application of IFCB, the dual-frequency IF satellite phase bias $\nabla b_{\Phi_{\text {dual }}}^{s}$ is on the right side of the equation above instead of the triple-frequency one in (10), which means stable float ambiguities can be expected. After convergence, the float WL ambiguities can be obtained. Similar to (8), the WL FCB corrections at one receiver can be obtained as

$$
\begin{aligned}
\widetilde{N}_{\mathrm{WL}_{\mathrm{L} 1 \mathrm{~L} 2}} & =\left\langle\frac{\nabla A_{\mathrm{WL}}}{\left(f_{1} /\left(f_{1}-f_{5}\right)\right) \lambda_{\mathrm{WL}_{\mathrm{L} 1 \mathrm{~L} 2}}}\right\rangle \\
\nabla b_{N_{\mathrm{WL}}}^{s} & =\nabla b_{P_{\text {dual }}}^{s}-\nabla b_{\Phi_{\text {dual }}}^{s}+b_{a} \\
& =\nabla A_{\mathrm{WL}}-\frac{f_{1}}{f_{1}-f_{5}} \lambda_{\mathrm{WL}_{\mathrm{L} 1 \mathrm{~L} 2}} \widetilde{N}_{\mathrm{WL}_{\mathrm{L} 1 \mathrm{~L} 2}} .
\end{aligned}
$$

The WL FCBs broadcasted to users is the average of WL FCBs in a network. With the WL FCB corrections, the integer property of WL ambiguities can be recovered. The integer WL ambiguity will be searched by LAMBDA method. It can be seen that the valid wavelength of the WL ambiguity $f_{1} /\left(f_{1}-f_{5}\right) \lambda_{\mathrm{WL}_{\mathrm{L} 1 \mathrm{~L} 2}}$ is $3.40 \mathrm{~m}$, which makes it much easier to obtain the WL ambiguities.

According to Geng and Bock [8] and Teunissen [16], the search area of ambiguity search space can be represented as

$$
S=\pi \chi^{2} \sqrt{|Q|} \approx \pi \chi^{2} \frac{\sigma_{L} \delta_{P}}{k \lambda_{1} \lambda_{2}},
$$

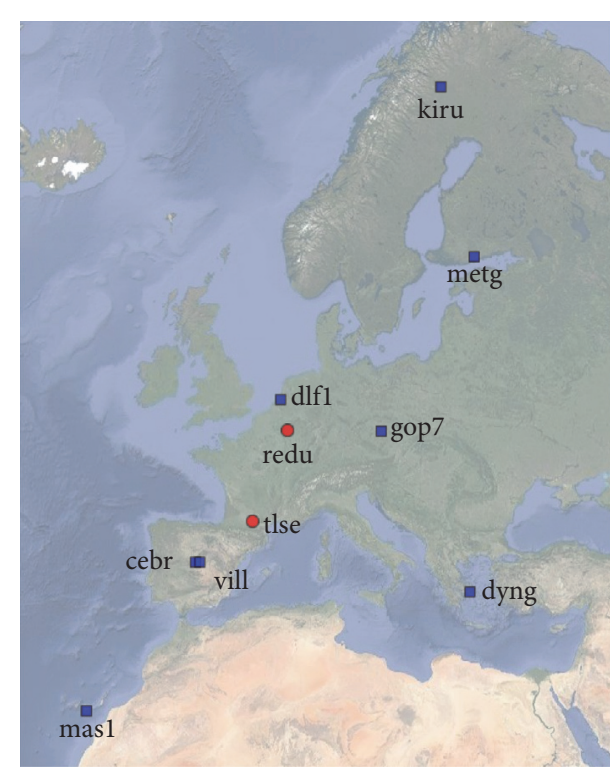

FIgURE 1: Distribution of observation stations.

where $S$ is the area of ambiguity search space, $\chi^{2}$ is the defined threshold, $|Q|$ is the determinant of float ambiguity variance-covariance matrix, and $\lambda_{1}$ and $\lambda_{2}$ are the carrierphase wavelength. It indicates that, with longer wavelength and smaller measurement noise, the ambiguities would be easier to be fixed. Although, for the triple-frequency IF carrier-phase, the noise is enlarged around 100 times, the valid wavelength for LAMBDA to be fixed is $3.40 \mathrm{~m}$, which makes it much easier to be fixed, compared to the situation in dual-frequency PPP where NL ambiguities need to be fixed.

\section{Tests and Results}

To test the validity of the proposed triple-frequency ambiguity resolution method, one sparse network with 10 stations in Europe where more than four GPS Block IIF satellites (transmitting the L5 signal) can be observed for more than 60 minutes is used. Among the 10 stations, 8 stations are used as reference stations to generate the single-difference IFCBs and FCBs while the other two stations are used as test user stations. PPP AR is implemented at the test user stations. All the datasets used in this work can be downloaded from the IGS Multi-GNSS Experiment (MGEX) website. Trimble R9 receiver is used in every station to receive triple-frequency signals. The distribution of 10 stations used is shown in Figure 1. The eight stations to generate IFCBs and FCBs are cebr, vill, dlf1, gop7, metg, kiru, dyng, and mas1, which are denoted in blue. The red stations redu and tlse are used to implement PPP AR. Dual-frequency PPP AR is first implemented in Section 4.1 using the same datasets as triple-frequency PPP AR in Section 4.2 to make a comparison. The same datasets are applied to fix the ambiguities of the same Block IIF satellites in both dual-frequency and triple-frequency PPP AR tests.

The observation span for redu and tlse is chosen on UTC May 9, 2015, from 02:35 am to 03:35 am when four Block IIF satellites (PRN 01, 03, 06, and 09) are in view simultaneously 
TABLE 1: Details of test (unit for distance is kilometer).

\begin{tabular}{lcccccc}
\hline Name & Interval (s) & Average distance & Min distance & Max distance & Number of Ref stations & Inter-Ref station distance \\
\hline redu & 1 & 1538 & 227 & 3006 & 8 & $35-4494$ \\
tlse & 1 & 1620 & 568 & 2913 & 8 & $35-4494$ \\
\hline
\end{tabular}

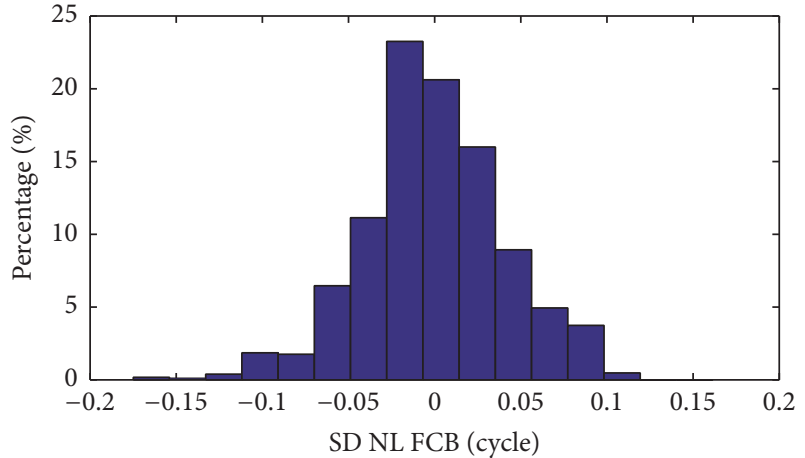

FIGURE 2: Histogram of the fractions of SD NL ambiguities after applying FCBs.

for all the stations mentioned above. The further details of the network can be found in Table 1. In both dual-frequency and triple-frequency processing, IGS final precise orbit and clock products are applied by both reference stations and test user stations. Single-difference is applied to eliminate the receiver contribution to biases in estimating the IFCBs, FCBs, and PPP AR implementation. In the tests, PRN 03 is set as the reference satellite due to high elevation. The datasets of reference stations are used to generate the FCBs to recover the integer property of ambiguities in dual-frequency processing. In triple-frequency processing, apart from the WL FCBs, IFCBs are first estimated to compensate the inconsistency in triple-frequency IF carrier-phase observations.

4.1. Dual-Frequency $P P P A R$. As illustrated in previous researches [2-4], the dual-frequency ionosphere-free ambiguities can be decomposed as WL and NL ambiguities. To recover the integer property of ambiguities, the WL and NL FCBs are first estimated by the reference stations. The WL ambiguities can be determined by MW combinations easily with a wavelength of $0.86 \mathrm{~m}$. With known coordinates of reference stations, the float ambiguities can be converged quickly. Therefore, the NL FCBs can be determined quickly as well. Shown in Figure 2 is the histogram of NL ambiguities after applying estimated NL FCBs. It can be seen that over $95 \%$ fraction parts of NL ambiguities fall into the -0.1 to 0.1 cycle after applying the estimated NL FCBs.

The one-hour observation at user test stations redu and tlse is divided into 2 30-minute sessions at each station. Due to short wavelength of narrow-lane combination, it takes tens of minutes for the test stations to fix the ambiguities. The positioning results for the four sessions at the test user stations are shown in Figure 2. It can be seen that, after successfully fixing the correct integer ambiguities, the positioning results are improved immediately. The time to first fix (TTFF) in these four sessions is $15,21,19$, and 29 minutes, respectively. The average TTFF is 21 minutes. For the two sessions at station redu, the RMS of horizontal positioning errors is reduced from $2.30 \mathrm{~cm}$ and $8.12 \mathrm{~cm}$ to $1.45 \mathrm{~cm}$ and $0.36 \mathrm{~cm}$ after fixing the ambiguities. The vertical RMS is reduced from $2.06 \mathrm{~cm}$ and $12.04 \mathrm{~cm}$ to $0.59 \mathrm{~cm}$ and $6.83 \mathrm{~cm}$. The positioning results for the two sessions at tlse station are improved as well. The horizontal errors are reduced from $5.47 \mathrm{~cm}$ and $3.05 \mathrm{~cm}$ to $0.76 \mathrm{~cm}$ and $0.53 \mathrm{~cm}$. As for the vertical direction, the error is reduced from $13.57 \mathrm{~cm}$ to $4.63 \mathrm{~cm}$ in the first session while the second session suffered slight degradation from $3.79 \mathrm{~cm}$ to $3.98 \mathrm{~cm}$.

The results of the dual-frequency PPP AR of GPS Block IIF satellites show that the dual-frequency PPP AR can achieve centimeter-level accuracy, but it takes long time to fix the ambiguities.

4.2. Triple-Frequency PPP AR. In triple-frequency PPP processing, dual-frequency IF code measurements of Block IIF satellites are applied instead of triple-frequency IF code measurements. This is because the triple-frequency IF combination would enlarge the noise about 100 times, which degrades the positioning results. Moreover, there is inconsistency between the triple-frequency IF code measurements and the dual-frequency based precise products. The bias between the dual-frequency and triple-frequency IF code measurements is shown in Figure 4.

It can be seen from Figure 3 that the biases between dualfrequency and triple-frequency IF code measurements fluctuate greatly. Therefore, it is more appropriate to apply dualfrequency IF code measurements. Unlike dual-frequency PPP, the IFCBs between triple-frequency and dual-frequency IF phase measurements need to be estimated to compensate the inconsistency between the triple-frequency IF carrierphase measurements and the IGS dual-frequency based precise orbit and clock precise products. The weighted IFCBs based on (12)-(14) are first calculated with the datasets of reference stations in the network. Shown in Figure 5 are the estimated weighted IFCBs with reference station datasets for PRN 01, PRN 06, and PRN 09 with PRN 03 as reference satellite and the first common epoch of each satellite as reference epoch. The initial single-difference $\operatorname{IFCB}\left(\delta_{0}\right)$ is set as 0 . From Figure 5, we can see that the variation of IFCB can be up to almost 3 meters over 2 hours which accounts for more than half a valid cycle $(3.4 \mathrm{~m})$ of WL L1/L2 in triple-frequency PPP AR. The aim of IFCB estimation is to obtain converged stable float ambiguities, which is the prerequisite to fix the ambiguities. Otherwise, the large variation IFCBs would contaminate the ambiguities, resulting in unstable float ambiguities which cannot be fixed.

As introduced before, the triple-frequency IF ambiguities can be decomposed as EWL ambiguities and WL ambiguities. 

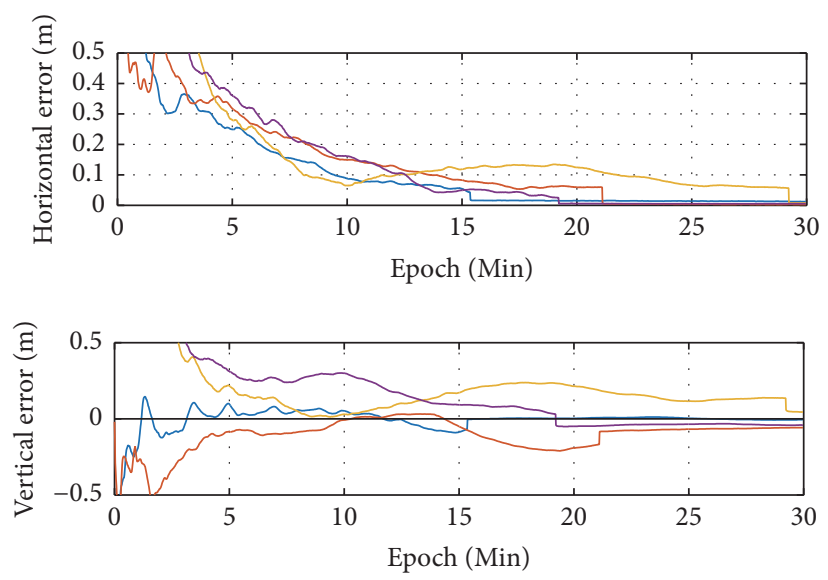

$\begin{array}{ll}\text { cebr Session } 1 & - \text { tlse Session } 1 \\ \text { cebr Session } 2 & \quad \text { tlse Session } 2\end{array}$

FIGURE 3: Dual-frequency positioning results of 4 30-minute sessions.
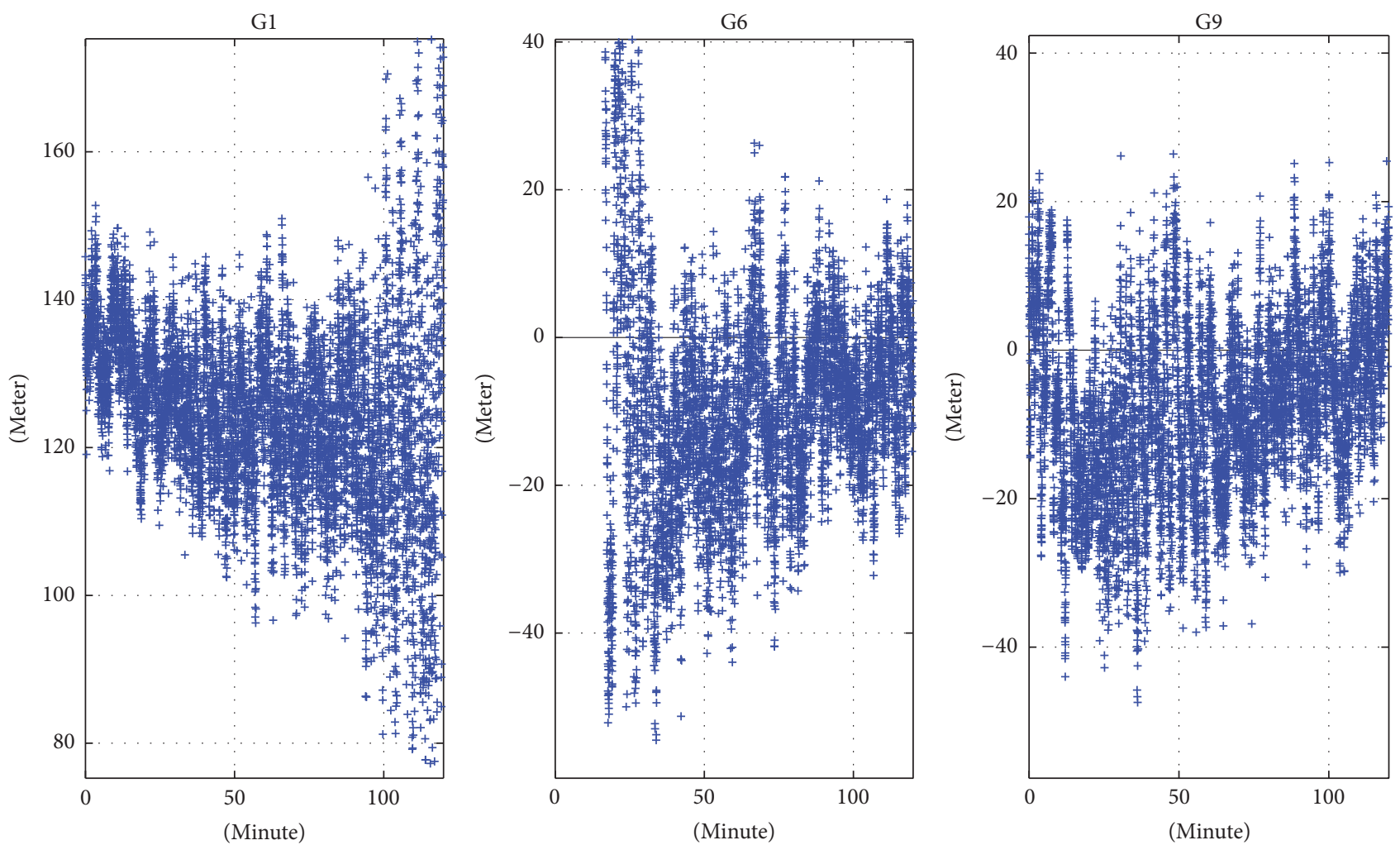

FIGURE 4: Bias between dual-frequency and triple-frequency IF code measurements.

After applying the correction of single-difference IFCBs and achieving stable single-difference float ambiguities, WL FCBs can be obtained after fixing the EWL ambiguities at reference stations with known coordinates. Single-difference EWL FCBs at reference station can be obtained by rounding the WL L2/L5 SD MW combinations directly due to large valid wavelength $(5.86 \mathrm{~m})$. Similarly, after the float ambiguities get converged and EWL ambiguities are fixed at reference stations, the WL FCBs can be obtained by rounding the converged WL ambiguities. Figure 6 shows that over 95\% fractional parts of WL ambiguities are within 0.1 cycles after applying the WL FCBs.

With estimated IFCBs, EWL FCBs, and WL FCBs, the triple-frequency PPP AR can be implemented at the test user stations redu and tlse. The cut-off angle is set as $15^{\circ}$ and an elevation-dependent weighted strategy was applied to measurements to reduce the impact of poor quality of measurements in low elevation. For EWL ambiguities, bias rounding 


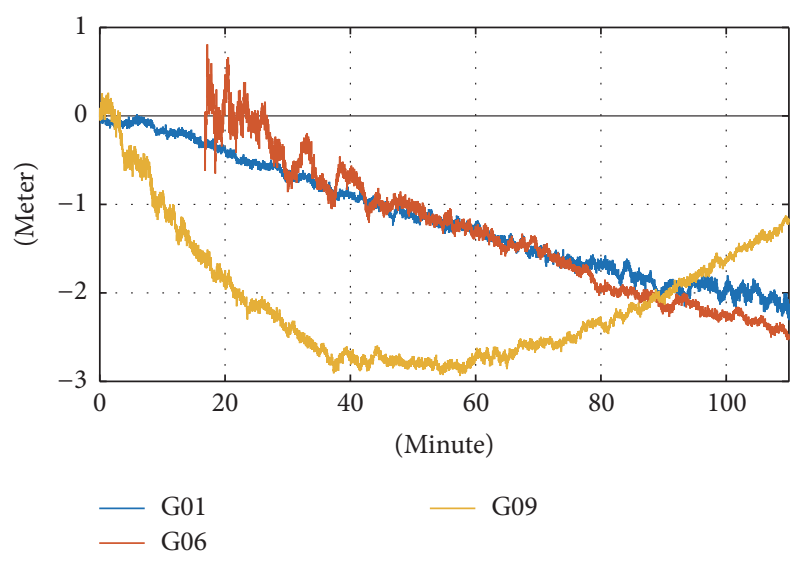

FIGURE 5: Reference epoch based SD IFCB.

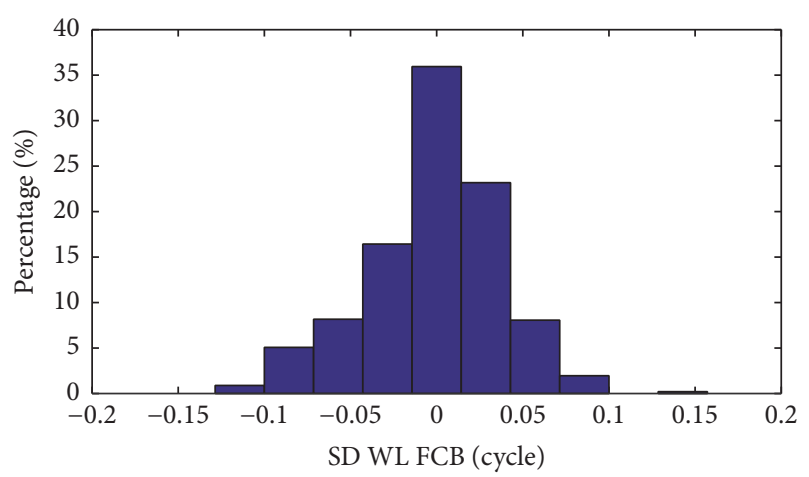

FIGURE 6: Histogram of the fractions of SD WL ambiguities after applying FCBs.

is directly applied due to the large wavelength of EWL. As for WL, LAMBDA method is applied to search for integer ambiguities and the ratio of the second minimum to the minimum quadratic form of residuals (a threshold of 3 ) is calculated to validate an ambiguity resolution. Because of the large noise of triple-frequency IF measurements, the weight of triple-frequency IF measurements is lower, compared to dual-frequency IF measurements. The weight ratio of dualfrequency IF phase measurements to triple-frequency IF phase measurements is about 33. Because of large noise of triple-frequency IF carrier-phase measurements and limited number of Block IIF satellites, the ambiguity resolution results can be expected that it could improve the results, but in limited extent.

As noted in the previous sections, the valid WL wavelength is about 3.4 meters, so ambiguity resolution is expected to be accomplished faster than conventional dual-frequency GPS PPP. With collected datasets at test user stations, onehour observation with four Block IIF satellites is utilized for triple-frequency PPP AR. SD EWL and SD WL FCBs can be obtained at reference stations to recover the integer property of ambiguities. In the first test, the one-hour observation at each station is divided into 10 sessions with 6 minutes in one session. In order to ensure the success of fixing ambiguities, ambiguities are attempted to be resolved after 3 minutes.
Figures 7 and 8 are the positioning errors in horizontal and vertical directions for the 10 sessions at redu and tlse, respectively. Each color represents positioning error of one session. Incorrect ambiguities are fixed for the last session at test user station tlse. It can be seen that, in most cases, the ambiguity resolution would have immediate impact on the positioning results. However, the positioning results still keep at decimeter level with slight improvement for some sessions. This is because only WL ambiguities are fixed in this work and the large noise of triple-frequency IF carrier-phase measurements and limited number of Block IIF satellites would contribute little to improving positioning results. To see the improvement of positioning results, the RMS of positioning errors after fixing ambiguities is listed in Table 2. The fixed solution results are compared with the float solution in the same period. It can be seen that correct triple-frequency WL ambiguities can be fixed in 19 sessions out of the 20 test sessions, except for the last session at station tlse. For the WL ambiguities fixed sessions at tlse, the positioning accuracy is slightly improved. The positioning accuracy after ambiguities fixed still keeps at decimeter level. For station redu, positioning accuracy degrades after WL ambiguity resolution in 4 sessions. The positioning accuracy keeps at decimeter level as well after WL ambiguity resolution. To further see the fast ambiguity resolution using triple-frequency measurements, the one-hour dataset is divided into 30 sessions with 2-minute observation in each session. The ambiguities are attempted to be fixed after 30 seconds of processing. The positioning results are shown in Table 3. Similarly, the positioning errors of fixed solution and float solution in the same period are compared. This time all the WL ambiguities can be fixed for all the 60 sessions at two test user stations. For station redu, the positioning accuracy degrades slightly after fixing the WL ambiguities in $40 \%$ sessions (12/30), while 2 sessions suffer positioning accuracy degradation at tlse. The fixed ambiguities of all sessions are correctly fixed, regardless of improvement of positioning or not. Therefore, the reason that leads to positioning degrading is not incorrect fixed ambiguities. There is great chance to obtain worse positioning results with limited number of WL ambiguities fixed partly due to the large noise of triple-frequency IF carrier-phase measurements. Besides, the quality of SD IFCBs and SD FCBs also has great impact on the positioning results.

\section{Conclusions}

In this paper, triple-frequency GPS PPP AR is investigated based on observed datasets. A general data processing method is proposed and verified. The collected datasets of triple-frequency IF carrier-phase measurements indicate an inconsistency with the IGS dual-frequency based precise clock product obtained by L1/L2 ionosphere-free combination. The interfrequency biases are estimated and applied to triple-frequency IF carrier-phase measurements in order to obtain relatively stable float ambiguities. Limited by the available triple-frequency datasets, one network with ten stations is utilized. In the network, the PPP AR is implemented at the two test user stations with the IFCBs and FCBs generated by eight reference stations. Compared with dual-frequency PPP 
TABLE 2: RMS of positioning results with fixed ambiguities and float ambiguities during the same period (after fixing ambiguities) for 10 6minute sessions at redu and tlse. In the table, "float" means RMS of positioning error with float ambiguities, "fixed" means RMS of positioning error with fixed WL ambiguities, "- " means there is no corresponding float solution RMS for comparison, and "fail" means failure of fixing correct WL ambiguities during the session. The unit in this table is centimeter.

\begin{tabular}{cccccccccccc}
\hline Station & 1 & 2 & 3 & 4 & 5 & 6 & 7 & 8 & 9 & 10 \\
\hline redu & & & & & & & & & & \\
$\quad$ Float & 33.5 & 41.4 & 43.1 & 43.1 & 56.9 & 49.1 & 62.1 & 69.8 & 60.9 & 54.5 \\
Fixed & 30.8 & 41.1 & 50.4 & 38.6 & 72.2 & 49.3 & 54.3 & 56.7 & 70.9 & 49.1 \\
\hline tlse & & & & & & & & & \\
Float & 45.8 & 63.1 & 97.6 & 81.3 & 89.0 & 125.8 & 106.0 & 61.8 & 98.0 & - \\
Fixed & 44.1 & 69.5 & 66.3 & 67.3 & 85.6 & 84.9 & 64.0 & 57.5 & 45.8 & Fail \\
\hline
\end{tabular}

TABLE 3: RMS of positioning results with fixed ambiguities and float ambiguities during the same period (after fixing ambiguities) for 30 2-minute sessions at redu and tlse. In the table, "float" means RMS of positioning error with float ambiguities and "fixed" means RMS of positioning error with fixed WL ambiguities.

\begin{tabular}{|c|c|c|c|c|c|c|c|c|c|c|}
\hline Station & 1 & 2 & 3 & 4 & 5 & 6 & 7 & 8 & 9 & 10 \\
\hline \multicolumn{11}{|l|}{ redu } \\
\hline Float & 58.2 & 68.6 & 68.6 & 59.2 & 96.7 & 86.7 & 94.3 & 67.6 & 76.2 & 51.6 \\
\hline Fixed & 58.1 & 41.3 & 61.9 & 48.3 & 53.5 & 68.7 & 76.8 & 70.3 & 51.9 & 44.8 \\
\hline \multicolumn{11}{|l|}{ tlse } \\
\hline Float & 132.8 & 54.1 & 88.8 & 132.7 & 91.6 & 80.1 & 118.8 & 173.0 & 146.7 & 97.1 \\
\hline Fixed & 89.1 & 44.1 & 80.7 & 123.8 & 88.3 & 59.5 & 55.2 & 113.9 & 92.2 & 73.5 \\
\hline Station & 11 & 12 & 13 & 14 & 15 & 16 & 17 & 18 & 19 & 20 \\
\hline \multicolumn{11}{|l|}{ redu } \\
\hline Float & 92.6 & 84.0 & 48.3 & 65.2 & 92.7 & 66.8 & 63.8 & 67.8 & 80.5 & 41.6 \\
\hline Fixed & 41.7 & 44.3 & 55.1 & 104.7 & 100.6 & 47.3 & 51.1 & 80.6 & 61.2 & 46.8 \\
\hline \multicolumn{11}{|l|}{ tlse } \\
\hline Float & 86.4 & 142.0 & 141.0 & 85.1 & 86.9 & 186.6 & 142.3 & 122.1 & 140.6 & 121.0 \\
\hline Fixed & 53.7 & 134.3 & 99.2 & 96.3 & 74.9 & 113.5 & 93.5 & 67.7 & 72.2 & 68.5 \\
\hline Station & 21 & 22 & 23 & 24 & 25 & 26 & 27 & 28 & 29 & 30 \\
\hline \multicolumn{11}{|l|}{ redu } \\
\hline Float & 60.8 & 78.5 & 87.9 & 55.5 & 64.9 & 57.9 & 76.7 & 58.3 & 120.6 & 51.8 \\
\hline Fixed & 74.8 & 75.9 & 96.9 & 59.4 & 62.0 & 80.5 & 103.4 & 61.9 & 62.2 & 85.8 \\
\hline \multicolumn{11}{|l|}{ tlse } \\
\hline Float & 102.2 & 80.4 & 75.0 & 159.9 & 123.9 & 161.0 & 100.1 & 163.8 & 160.9 & 162.4 \\
\hline Fixed & 57.8 & 78.2 & 39.3 & 99.6 & 44.7 & 51.2 & 52.8 & 59.8 & 249.8 & 108.8 \\
\hline
\end{tabular}

AR, triple-frequency ambiguities can be fixed much faster. However, the fixed triple-frequency solution still keeps at decimeter level. In some cases, the accuracy even degrades after correctly fixing the ambiguities, because only a limited number of WL ambiguities are fixed and the triple-frequency IF combination enlarges the measurement noise. The test results show that most positioning results become better after fixing ambiguities, which confirms the effectiveness of the proposed fast triple-frequency PPP AR method. In this study, the satellite L5 PCO and PCV are assumed as the same value of satellite $\mathrm{L} 1$ and $\mathrm{L} 2$. The receiver L5 PCO and PCV are assumed to be the same value of L2 due to similar frequency. Actually, due to the large coefficients in triplefrequency IF combination, the effect of PCO and PCV could have a decimeter-level impact on the measurements in some situations, which may cause inaccurate estimation of IFCBs. The estimation of IFCBs can be omitted if triple-frequency precise clock product is available in the future, and the results can be better if precise information (e.g., satellite and receiver $\mathrm{PCO}$ and PCV) for the third frequency signal can be available. 

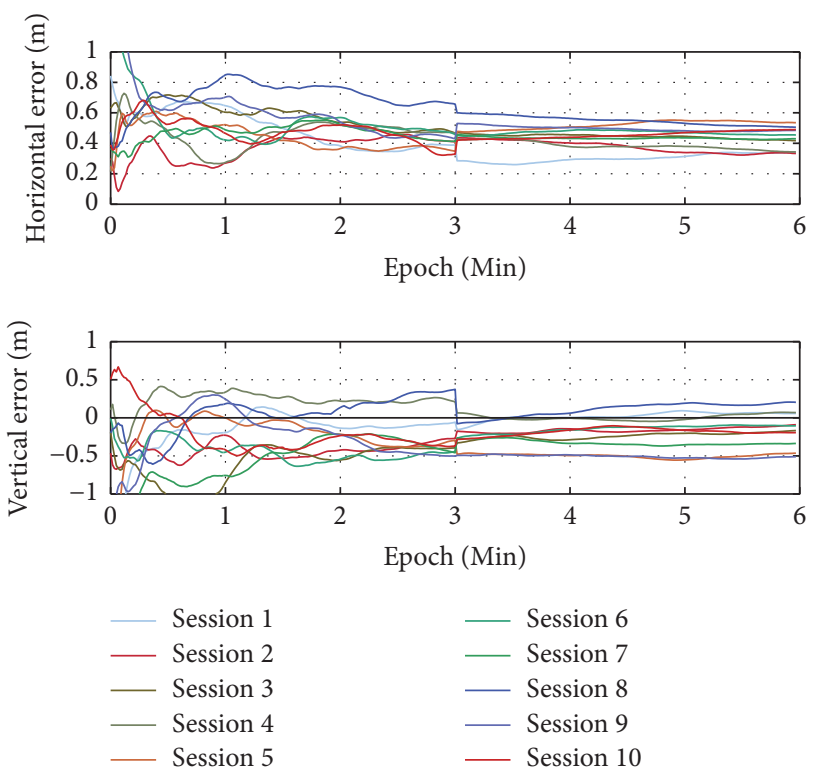

Figure 7: Positioning results of 10 6-minute sessions at redu.
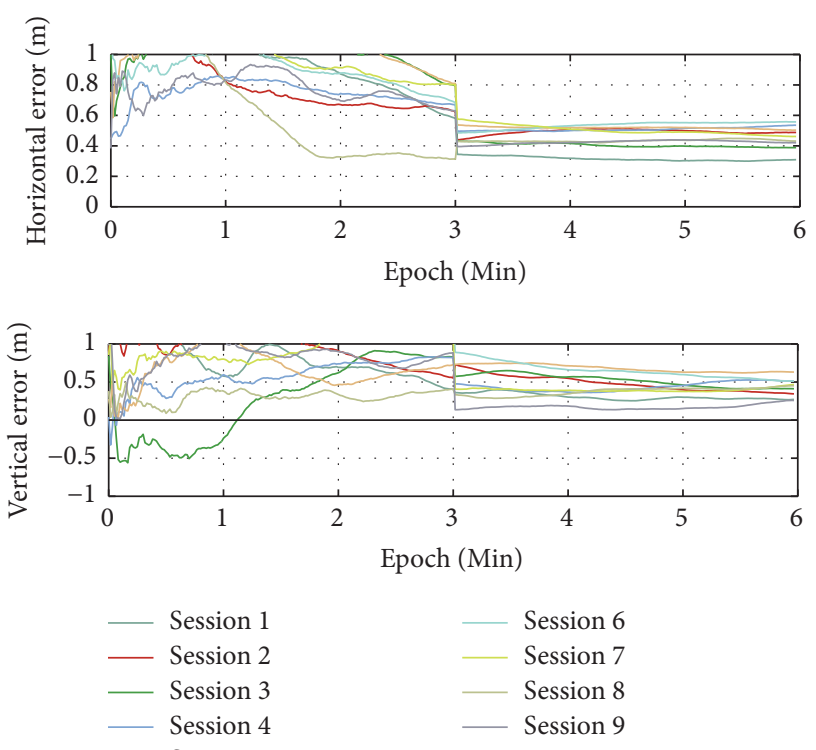

FIgURE 8: Positioning results of 10 6-minute sessions at tlse.

\section{Competing Interests}

The authors declare that there is no conflict of interests regarding the publication of this paper.

\section{References}

[1] J. F. Zumberge, M. B. Heflin, D. C. Jefferson, M. M. Watkins, and F. H. Webb, "Precise point positioning for the efficient and robust analysis of GPS data from large networks," Journal of Geophysical Research B: Solid Earth, vol. 102, no. 3, pp. 50055017, 1997.

[2] P. Collins, "Isolating and estimating undifferenced GPS integer ambiguities," in Proceedings of the Institute of Navigation
National Technical Meeting (NTM '08), pp. 720-732, San Diego, Calif, USA, January 2008.

[3] M. Ge, G. Gendt, M. Rothacher, C. Shi, and J. Liu, "Resolution of GPS carrier-phase ambiguities in Precise Point Positioning (PPP) with daily observations," Journal of Geodesy, vol. 82, no. 7, pp. 389-399, 2008.

[4] D. Laurichesse, F. Mercier, J.-P. Berthias, P. Broca, and L. Cerri, "Integer ambiguity resolution on undifferenced GPS phase measurements and its application to PPP and satellite precise orbit determination," Navigation, Journal of the Institute of Navigation, vol. 56, no. 2, pp. 135-149, 2009.

[5] W. G. Melbourne, “The case for ranging in GPS-based geodetic systems," in Proceedings of the 1st International Symposium on Precise Positioning with the Global Positioning System, vol. 1519, pp. 373-386, Rockville, Md, USA, April 1985.

[6] G. Wübbena, "Software developments for geodetic positioning with GPS using TI-4100 code and carrier measurements," in Proceedings of the 1st International Symposium on Precise Positioning with the Global Positioning System, vol. 19, Rockville, Md, USA, April 1985.

[7] J. Geng, F. N. Teferle, X. Meng, and A. H. Dodson, "Towards PPP-RTK: ambiguity resolution in real-time precise point positioning," Advances in Space Research, vol. 47, no. 10, pp. 1664-1673, 2011.

[8] J. Geng and Y. Bock, "Triple-frequency GPS precise point positioning with rapid ambiguity resolution," Journal of Geodesy, vol. 87, no. 5, pp. 449-460, 2013.

[9] S. Gu, Y. Lou, C. Shi, and J. Liu, "BeiDou phase bias estimation and its application in precise point positioning with triplefrequency observable," Journal of Geodesy, vol. 89, no. 10, pp. 979-992, 2015.

[10] M. Wang, Ambiguity resolution with precise point positioning [Ph.D. thesis], Department of Geomatics Engineering, University of Calgary, 2014.

[11] D. Laurichesse, Handling the Biases for Improved TripleFrequency PPP Convergence, GPS World, 2015.

[12] H. Li, X. Zhou, B. Wu, and J. Wang, "Estimation of the interfrequency clock bias for the satellites of PRN25 and PRN01," Science China: Physics, Mechanics and Astronomy, vol. 55, no. 11, pp. 2186-2193, 2012.

[13] O. Montenbruck, U. Hugentobler, R. Dach, P. Steigenberger, and A. Hauschild, "Apparent clock variations of the Block IIF-1 (SVN62) GPS satellite," GPS Solutions, vol. 16, no. 3, pp. 303-313, 2012.

[14] P. J. G. Teunissen, “The least-squares ambiguity decorrelation adjustment: a method for fast GPS integer ambiguity estimation," Journal of Geodesy, vol. 70, no. 1-2, pp. 65-82, 1995.

[15] Y. Feng, "GNSS three carrier ambiguity resolution using ionosphere-reduced virtual signals," Journal of Geodesy, vol. 82, no. 12, pp. 847-862, 2008.

[16] P. J. G. Teunissen, "On the sensitivity of the location, size and shape of the GPS ambiguity search space to certain changes in the stochastic model," Journal of Geodesy, vol. 71, no. 9, pp. 541551, 1997. 


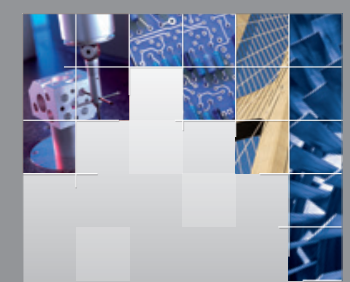

\section{Enfincering}
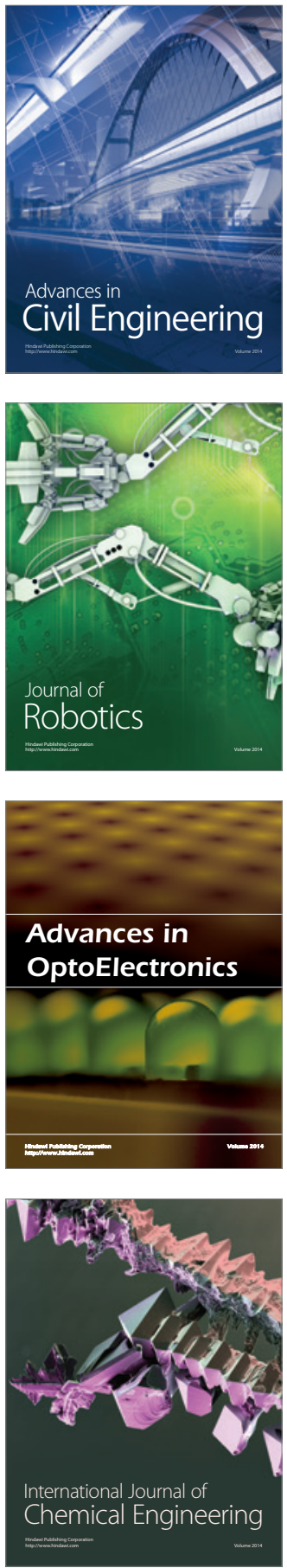

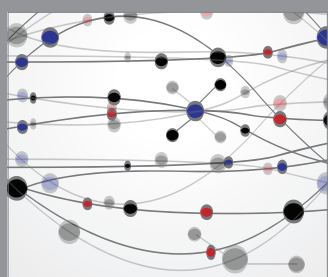

The Scientific World Journal

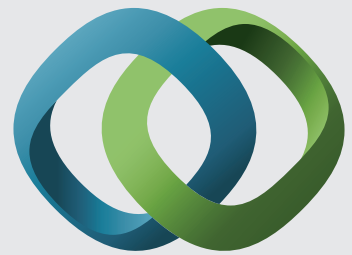

\section{Hindawi}

Submit your manuscripts at

https://www.hindawi.com
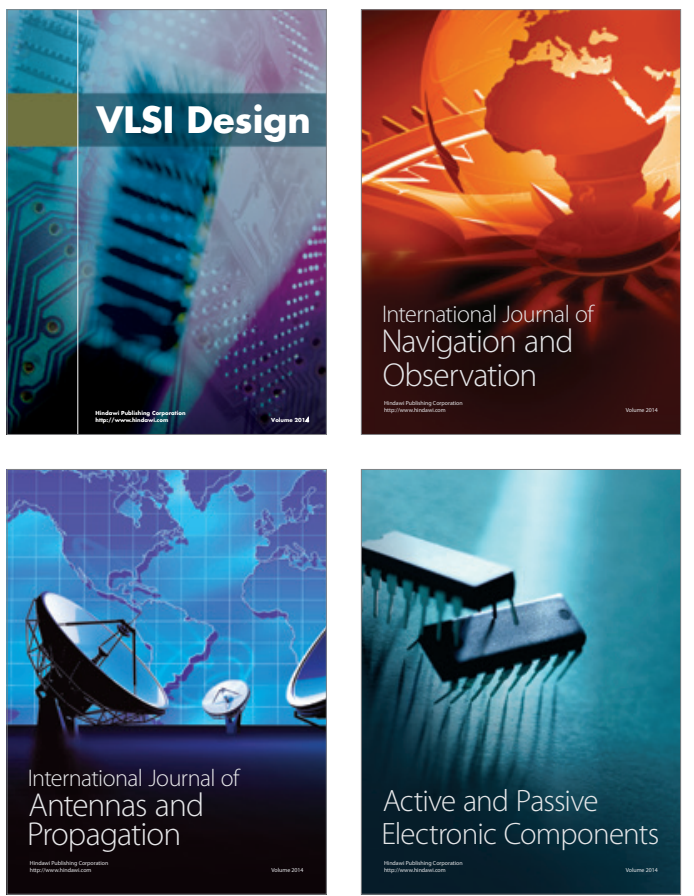
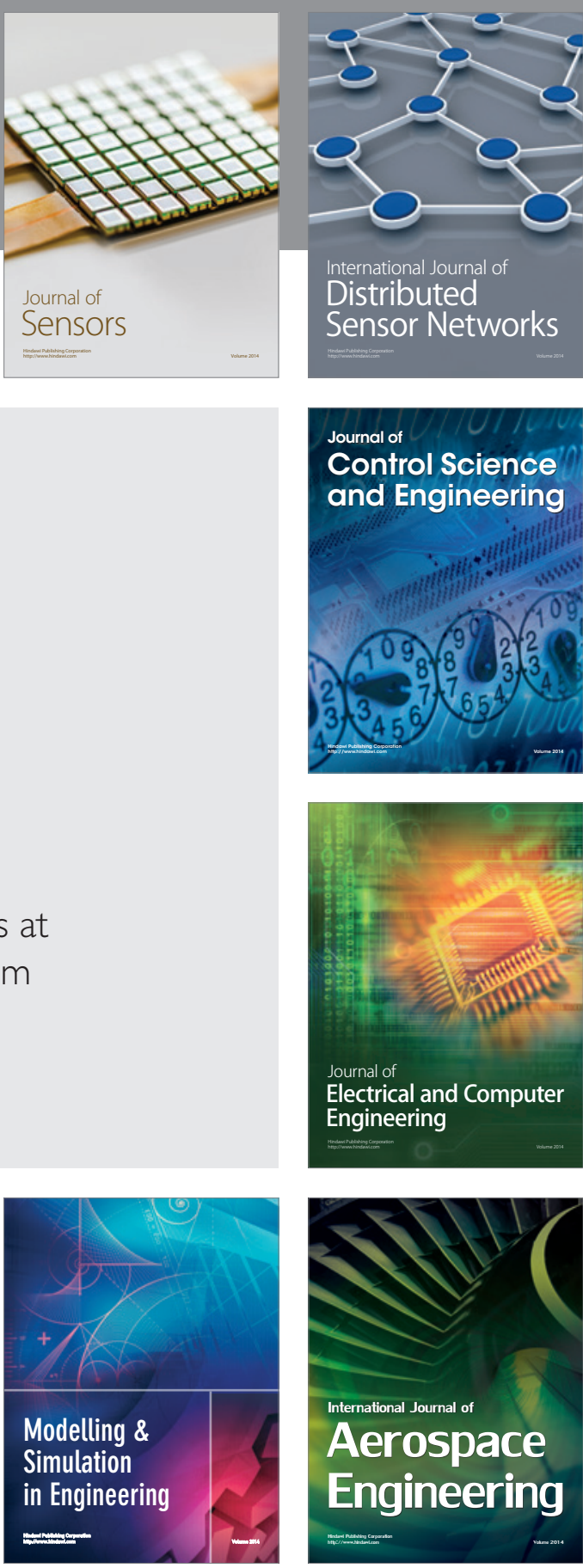

International Journal of

Distributed

Sensor Networks

$-$

Joumal of

Control Science

and Engineering
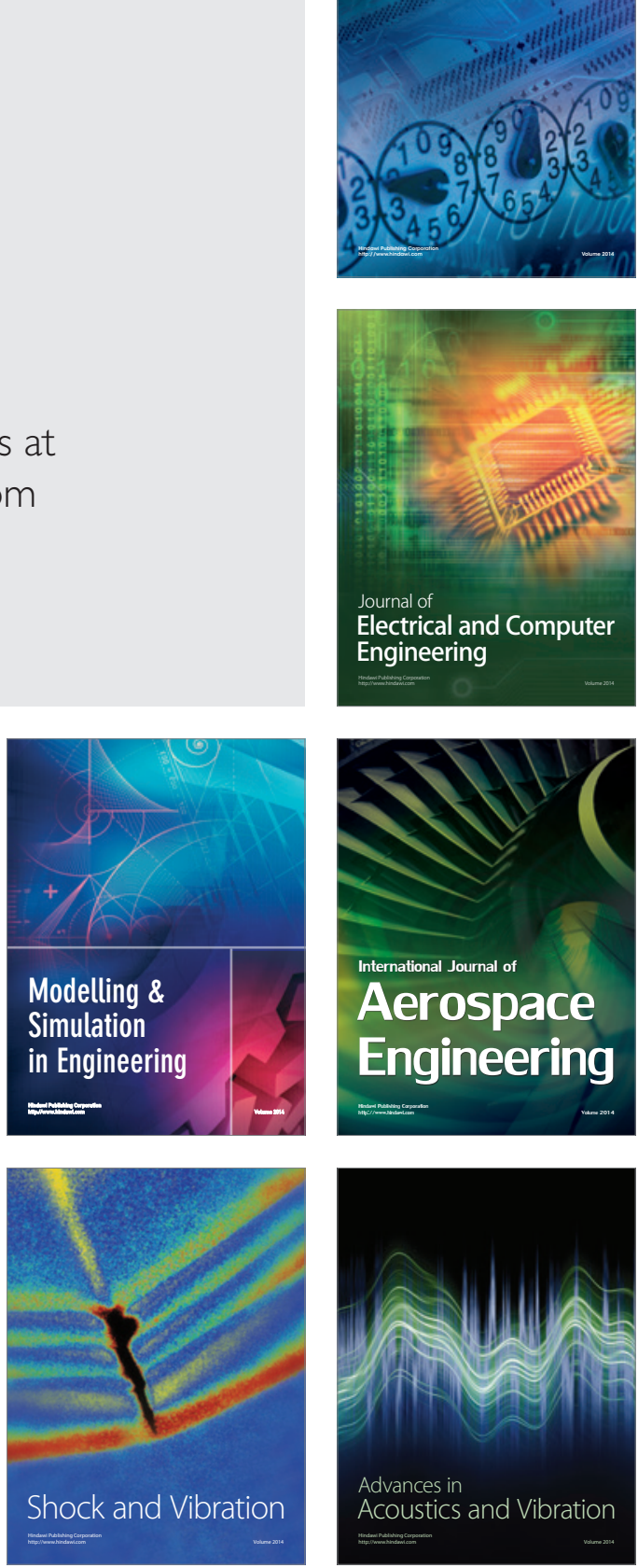